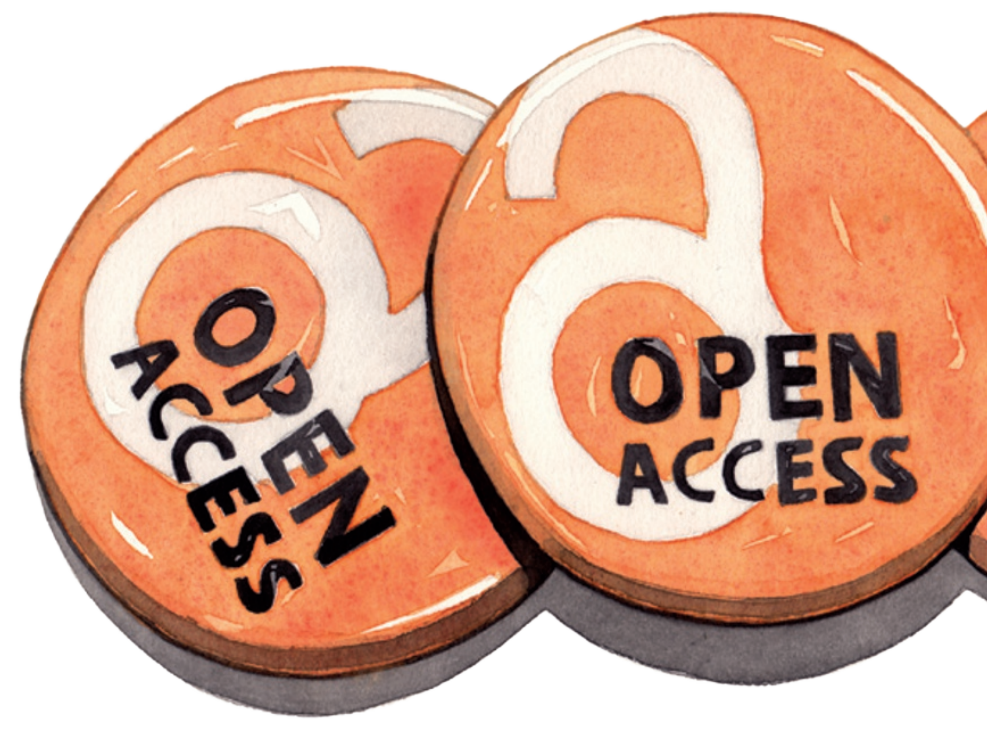

\section{How to hasten}

\section{open access}

Three advocates for a universally free scholarly literature give their prescriptions for the movement's next push, from findability to translations.

\section{ALMA SWAN \\ Time to align policy}

Director of European advocacy, Scholarly Publishing and Academic Resources Coalition (SPARC) Europe

Policies for open-access publishing have made rapid progress, raising a pressing issue - if the research literature is to become completely open within the next 5 years, different policies must be aligned. For researchers who are funded by more than one source, this harmonization is crucial in creating a single, simple path to making their work open access, affordably and sustainably.

Funders, universities, nations and states need to work together now to secure good open-access policy. What does that look like? It is clear, accurate and focused. It supports new models for research communication. It does not let copyright become a hurdle and does not interfere with authors' choice of journals. It mandates immediate access. It does not perpetuate a broken market, but finds ways to curb costs.

Good policy doesn't refer to 'gold' open

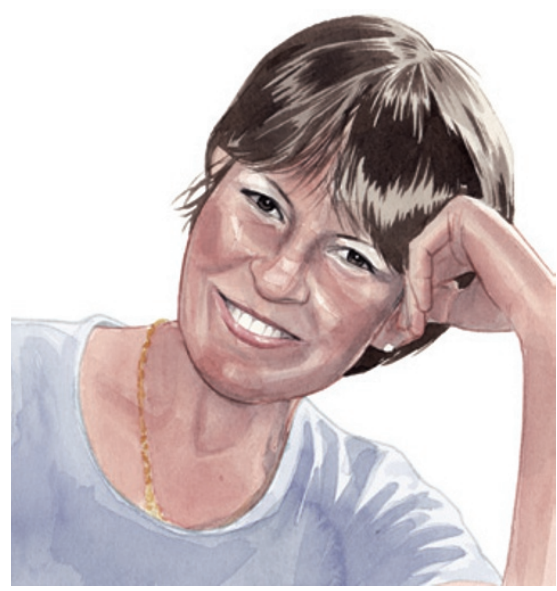

access as 'author pays' or 'paid for', because $66 \%$ of open-access journals do not charge. It uses the proper definition: journals that make their content immediately and freely available on the Internet. It doesn't call 'green' open access 'embargoed access', because $60 \%$ of the time it is not. It defines it as literature that is made open access directly by the author, usually through a repository. It doesn't assume that green open access harms publishers, because evidence shows that it does not.

Good policy exploits the fact that gold and green routes can both provide articles with licences that maximize re-use (see page 440).
It introduces measures of compliance and sanctions for non-compliance - because these shape behaviour. It embraces the fact that open access will cause disruptive change. And it puts the interests of research, and of the public that pays for it, before all others.

Specifically, funders and universities must work together to align policy with respect to permissible embargoes, timing of repository deposits for green open access and whether research grants can be used to buy gold open access.

They must consider whether payments can be made to 'hybrid' journals (subscription journals that make articles open access for a fee) and the position that funders take on rights, licensing requirements and which repositories can be used.

The European Commission (EC) has been explicit about its intentions on these details. From January 2014, it will require articles to be placed in institutional repositories immediately upon publication or acceptance. It permits only a six-month embargo in disciplines within science, technology, engineering and medicine, and a 12-month embargo in the humanities and social sciences. The policy encourages authors to retain copyright and to give publishers licences to publish their work, and it allows grant funds to be used for publishing charges. The EC has stated its hope that European countries will develop open-access policies in line with its own.

State-level policy proposals in the United States align with proposed federal policy in terms of permitted embargo times (the maximum is 12 months). In Belgium and Ireland, funder and university policies are aligning their focus on deposits in repositories, requiring this for research assessment and echoing the EC's maximum permitted embargo times. In Spain, Norway and Denmark, policies that align with that of the EC are already in place. The two Australian funders' policies are similar, calling for deposit in repositories and differing only slightly on permitted embargoes.

So, harmonization is happening. But the development of seamless policies among funders needs to be the focus of advocacy. It will mean less author confusion and greater compliance. Authors will begin to understand the potential of truly open research and be inspired to devise further innovative practices. Then we can expect true disruption: a very different kind of scholarly communication, catalysed by good policy.

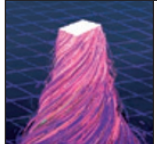

THE FUTURE OF PUB ISSHNG A Nature special issue. nature.com/scipublishing 


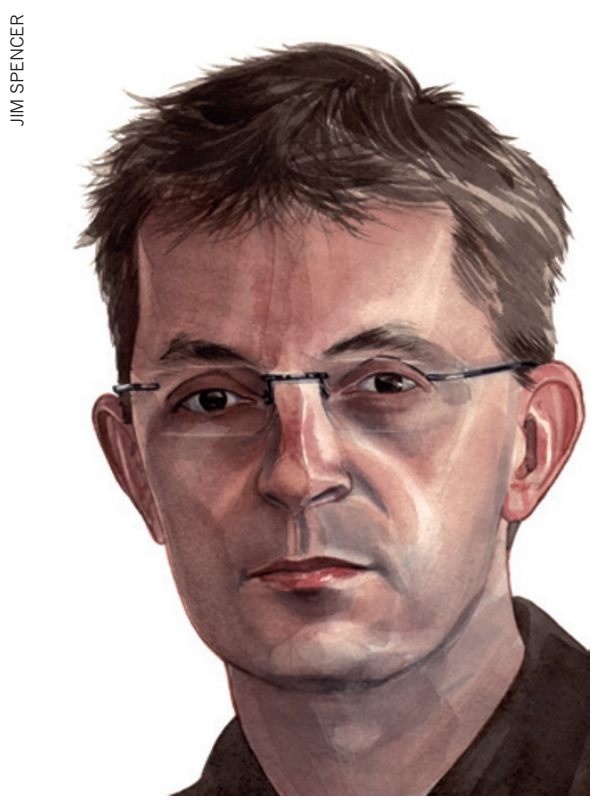

MATTHEW COCKERILL Make indexing fast and fair

\section{Managing director, BioMed Central}

Imagine a world in which Google demands that websites prove their worth for several years before their pages become searchable. In essence, that is the model that is currently in place for scientific publishing. For a journal to be indexed within PubMed Central, Web of Science and Scopus - in other

words, for its publications to be easily visible to scientists - it must provide a track record of high-quality publications. The catch-22 is that while proving itself, that journal cannot offer authors one of their most important requirements: discoverability.

For open access to take flight, the indexing and visibility of new journals needs sorting out. PubMed Central's original policy of rapid inclusion of open-access journals was crucial in helping the model to prove itself. The retreat in 2009 from this inclusiveness has threatened to stymie evolution in science publishing, helping incumbents to defend their position and disadvantaging some new entrants.

The controversy generated when the journal eLife jumped the queue by appearing in PubMed Central before having published a single paper, let alone the current usual requisite of at least 15 , highlights how baffling entry criteria have become. It would be a disservice to the community for a high-quality journal such as eLife not to be promptly tracked. But the jump-start gave the unhealthy impression that, to paraphrase George Orwell, 'all journals are treated equally, but some are treated more equally than others'.
Conversely, one of the leading journals we publish at BioMed Central, Genome Medicine, has only just been added to Web of Science by Thomson Reuters 4 years after its launch. The delay occurred despite a strong editorial board, a steady publication rate of around 90 articles a year and a predicted impact factor of around 6 - greater than those for $95 \%$ of the journals included in Web of Science.

'Predatory publishers' - those offering journals of low quality - are undoubtedly a concern (see page 433). But endlessly raising the walls of the citadel, or making those walls scalable only by journals with friends in high places, is not the answer: it slows scientific progress.

Instead, indexing services should err on the side of including content from any new journal that meets basic standards, perhaps flagging it with a 'provisional' tag, but ensuring that it is discoverable and citable. If journals are subsequently found to have poor editorial standards or excessively low citation rates, they can easily be dropped from the index, and prior content removed or flagged with a warning.

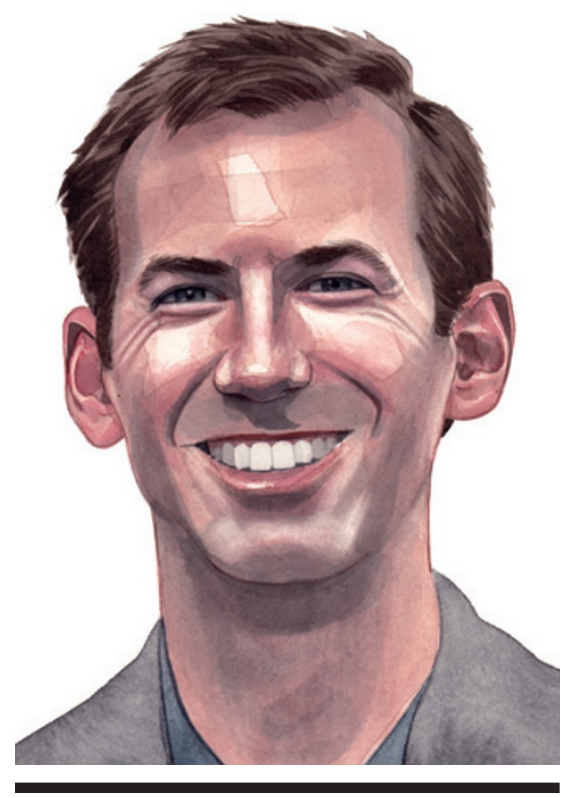

DOUGLASSIPP

\section{Translate local journals}

\section{RIKEN Center for Developmental Biology, Kobe, Japan}

Once open-access publishing becomes common practice, citizens of English-speaking countries will enjoy the unearned advantage of being able to read and use research funded by other nations. But those nations' populations may be unable to read articles reporting research funded by their own taxes.

Governments and public-funding agencies in such nations will soon find themselves pressed to resolve this inequity.

In the major east Asian scientific powers of China, Japan and South Korea - the world's 2nd, 4 th and 12th most-productive nations in terms of numbers of research publications - research and funding institutions have established open repositories for published research. The National Science Library of the Chinese Academy of Sciences, for example, mandated in 2009 that its members file articles in its open repository within one month of publication. At present, this seems to be used mainly for articles in Chinese-

\section{"The solution will be costly and time- consuming."}

language journals. Japan's largest funder of basic scientific research, the Ministry of Education, Culture, Sports, Science and Technology (MEXT), proposed only last year that researchers receiving MEXT grants should report the access status of their publications. It has not specified any access requirements.

Japan and South Korea have both invested in science communication and public-understanding efforts to improve their citizens' access to scientific information, through better funded science museums, media and online programmes and training of communication and outreach specialists. For example, in 2001 Japan opened a large science museum, the Miraikan, and there are now programmes in science communication and journalism at Hokkaido and Waseda universities. South Korea has increased funding for its national science museum, and the Korean Academy for Science and Technology in Seongnam now offers media training.

But these activities fall short of unmediated access to full sets of data, methods and analyses. Local-language journals, another partial solution, remain uneven in quality and low in impact. English is still undisputedly the common language of the sciences.

The clearest way to achieve true open access in non-English speaking nations might be to borrow models from the openaccess movement itself. A green open-access approach to lowering the language barrier could see funders call for scientists or institutions to produce local language translations of any published work conducted using public funds and host them in open repositories. A gold open-access approach, by contrast, might subsidize translations by journal publishers or other third parties. In either case, the solution will be costly and time-consuming, and require countries where English is not the native language to work harder than their anglophone counterparts to ensure public access to one of humanity's most precious resources - its knowledge.

M.C. has declared competing financial interests: see go.nature.com/ns5f9q for details. 